\title{
Elderly patients with stage III NSCLC survive longer when chemotherapy is added to radiotherapy-fortune favours the bold
}

\author{
Surein Arulananda ${ }^{1,2,3}$, Paul Mitchell ${ }^{1,3,4}$ \\ ${ }^{1}$ Cancer Immuno-Biology Laboratory, Olivia Newton-John Cancer Research Institute, Heidelberg, Australia; ${ }^{2}$ School of Cancer Medicine, La Trobe \\ University, Heidelberg, Australia; ${ }^{3}$ Department of Medical Oncology, Austin Health, Heidelberg, Australia; ${ }^{4}$ Department of Medicine, University of \\ Melbourne, Parkville, Australia \\ Correspondence to: Paul Mitchell, MBChB, MD. Department of Medical Oncology, Austin Health, 145 Studley Road, Heidelberg 3084, Victoria, \\ Australia. Email: Paul.MITCHELL@austin.org.au. \\ Comment on: Miller ED, Fisher JL, Haglund KE, et al. The Addition of Chemotherapy to Radiation Therapy Improves Survival in Elderly Patients \\ with Stage III Non-Small Cell Lung Cancer. J Thorac Oncol 2018;13:426-35.
}

Submitted Aug 15, 2018. Accepted for publication Aug 21, 2018.

doi: $10.21037 /$ tlcr.2018.08.12

View this article at: http://dx.doi.org/10.21037/tlcr.2018.08.12

Around a third of non-small cell lung cancer (NSCLC) patients present with stage III disease. The majority are not suitable for surgery and until very recently, the standard-ofcare for unresectable, good performance status patients with stage III disease has been platinum-doublet chemotherapy with high dose radiotherapy (RT), with no additional treatment. What is less clear is the benefit of aggressive treatment with curative intent in elderly patients, who are poorly represented in clinical trials. The median age at diagnosis with lung cancer is 70 years (1) which is also the age considered elderly as this is when the majority of senescent-related changes occur (2). A recent paper from Miller et al. in the Fournal of Thoracic Oncology supports combined chemotherapy and RT in elderly patients with stage III NSCLC (3). However, the advent of effective consolidation immune checkpoint inhibition may offer an opportunity to extend effective treatment to a greater proportion of elderly patients.

The treatment of stage III NSCLC is based on a series of clinical trials, which generally recruited fit younger patients. Combining chemotherapy with radiotherapy (CRT) gave superior survival to RT alone with randomised trial data for both concurrent and sequential approaches (4-6). The landmark RTOG 9410 phase III study randomised 610 patients with stage II or III unresectable NSCLC to two different regimens of concurrent chemoradiation (CCRT) with cisplatin and either etoposide or vinblastine versus sequential chemoradiation (SCRT) with cisplatin and vinblastine followed by RT (7). The 5-year overall survival (OS) was significantly higher for patients treated with the concurrent regimens compared to sequential (16\% and $13 \%$ versus $10 \%, \mathrm{P}=0.046)$. Of note, the median age of patients in this important study was 61 years.

Subsequently a meta-analysis evaluating six randomised clinical trials confirmed an improvement in 5 -year OS from $10.6 \%$ with SCRT compared to $15.1 \%$ with CCRT [hazard ratio (HR) $=0.84$; 95\% CI, 0.74-0.95, P=0.004] (8). There was a statistically significant reduction in locoregional progression with CCRT, but not distant progression. However, CCRT was associated with greater toxicity, especially grade $\geq 3$ acute oesophagitis as high as $18 \%$ compared to $4 \%$ of patients with SCRT $(\mathrm{P}<0.001)$, but reassuringly pneumonitis rates were similar.

The three commonly used CCRT regimens in the clinic are cisplatin and etoposide $(9,10)$, carboplatin and paclitaxel $(11,12)$ and cisplatin with pemetrexed (13). A phase III randomised trial in a Chinese population comparing 60-66 Gy RT in combination with either cisplatin and etoposide or carboplatin and paclitaxel in 200 patients with stage III NSCLC demonstrated a greater landmark 3-year OS of $41.1 \%$ with the cisplatin regimen versus $26.0 \%$ with the carboplatin regimen $(\mathrm{P}=0.024)(14)$. The tradeoff however was increased toxicities, with a higher rate of grade $\geq 3$ oesophagitis ( $20.0 \%$ with cisplatin-etoposide versus $6.3 \%$ with carboplatin-paclitaxel, $\mathrm{P}=0.009$ ). The carboplatin-paclitaxel regimen remains widely used in 
clinical practice especially in older patients with borderline performance status. We previously reported a retrospective series from our institution where of 75 stage III NSCLC patients treated with CCRT (44 with cisplatin-etoposide and 31 with carboplatin-paclitaxel), there was no difference in OS (13.7 months with cisplatin-etoposide versus 20.7 months with carboplatin-paclitaxel, $\mathrm{P}=0.989)(15)$. In line with the literature, grade $\geq 3$ toxicity rates (neutropenia, thrombocytopenia) were higher with the cisplatin-based combination. Therefore, carboplatin-paclitaxel based CCRT is not only a reasonable option for fit patients, but also for those considered unfit for cisplatin, with preexisting hearing impairment, poor baseline renal function or significant co-morbidities.

A number of attempts have been made to improve on CCRT, including several trials of consolidation chemotherapy which failed to improve OS, but with significant toxicity (16). Neo-adjuvant chemotherapy given prior to CCRT similarly failed to improve outcomes (17), and despite a high rate of failure in the brain, prophylactic cranial irradiation did not improve OS (18). Another failed strategy was from the RTOG 0617 study, a phase III $2 \times 2$ factorial designed study, which showed that increasing the dose of RT to 74 Gy compared to standard $60 \mathrm{~Gy}$ with or without the addition of concurrent cetuximab to carboplatin-paclitaxel chemotherapy, not only failed to translate into an OS benefit, but resulted in harm, while the addition of cetuximab to carboplatin-paclitaxel chemotherapy did not result in an improvement in survival (19). The outcomes of this study were a great disappointment and suggested that changing chemotherapy and RT doses and schedules were unlikely to substantially impact on patient outcomes.

Against this backdrop, the PACIFIC study has revolutionised the management of stage III NSCLC patients. The addition of 12 months of durvalumab, an antiprogrammed death ligand 1 (PD-L1) antibody following CCRT improved the median progression-free survival (PFS) from 5.6 months (95\% CI, 4.6-7.8) to 16.8 months (95\% CI, 13.0-18.1), HR 0.52 (95\% CI, 0.42-0.65), P<0.001 (20). A recent press-release confirmed that the study met its OS end-point and will be presented at the plenary session at the World Conference of Lung Cancer in Toronto, Canada in September 2018. Of note, only patients who had received CCRT, not SCRT, were included in the study, and the magnitude of benefit has led to this agent being rapidly incorporated into clinical practice.

The median age of patients recruited to the PACIFIC trial was 65 , substantially younger than the median age at presentation for the population of 70 years (20). Many other important trials recruited even fewer elderly patients making it challenging to extrapolate clinical trial results to the optimal management of elderly patients, which form a large proportion of patients seen in the clinic. As age is a less than ideal method of determining the best treatment for patients, certain factors and tools have been proposed, the most established being the Comprehensive Geriatric Assessment (CGA) (21). It includes a multi-domain method of screening elderly patients, including medical comorbidities, mental health, functional capacity, social supports and environmental factors. This validated tool can assist with decision-making on treatment options, and hopefully avoid medical practitioners under or over-treating patients.

What are the data we have available to guide us in treating the elderly with stage III NSCLC? It is of concern that elderly patients meeting the eligibility requirements for enrolment in many prospective clinical trials may not be representative of the elderly NSCLC population. A retrospective study determined that high dose RT at $60 \mathrm{~Gy}$ was safe and resulted in a similar 5 -year survival for patients aged 75 years and over compared to younger patients (22). A number of subsequent retrospective analyses of the outcomes in elderly patients treated with CCRT for stage III NSCLC showed that toxicity rates were higher (hematologic and pneumonitis) but survival outcomes were similar to the non-elderly cohort (23-26).

The first and, to our knowledge, only prospective randomised clinical trial designed to specifically address this question was run by the Japanese Clinical Oncology Group which recruited 200 patients with unresectable stage III NSCLC over the age of 70 years to CRT (60 Gy plus concurrent low-dose daily carboplatin) versus RT alone (27). The study found that the median OS was 22.4 months (95\% CI, 16.5-33.6) for CCRT versus 16.9 months with RT (95\% CI, 13.4-20.3), HR 0.68, $95 \%$ CI, $0.47-0.98, \mathrm{P}=0.0179$. In keeping with the other studies, grade $\geq 3$ haematological toxicity rates were higher with CCRT, while the pneumonitis rates were similar at 3-4\%. The main criticisms of this study were that Asian-only patients were recruited, making it difficult to extrapolate these data to the non-Asian population especially in the context of a phase III study conducted in the United States showing that single agent carboplatin in addition to RT did not result in an OS benefit (28) and that platinum-doublet chemotherapy is considered standard-of-care in fit patients 
regardless of age. In fact, in their consensus paper, the EORTC Elderly Task Force and International Society for Geriatric Oncology (SIOG) acknowledges these limitations of the study and recommends that CCRT be offered to elderly patients, so long as their performance status and comorbidities are acceptable (29).

In light of the paucity of data comparing CRT versus RT in the elderly population, Miller et al. interrogated the National Cancer Database (NCDB), a United States based data registry across 1,500 sites, to retrospectively study real-world outcomes (3). They defined elderly as 70 years old and over and included patients from 2003-2014 who had received $\geq 59.4$ Gy RT with or without concurrent or sequential chemotherapy. The investigators identified 5,023 elderly patients treated with RT alone and 18,206 patients treated with CRT, making this the largest comparison series in stage III NSCLC to date. Not unexpectedly, CRT patients had statistically significant less comorbidities and were younger, 75.8 versus 79.4 years old. Among the CRT patients, $87 \%$ received CCRT and $13 \%$ SCRT.

The key finding from the study was that the median OS after propensity score-matching was 17.2 months (95\% CI, 16.6-17.8) for patients treated with CRT and 12.2 months (95\% CI, 11.8-12.6) for patients treated with RT (HR $=0.67,95 \%$ CI, 0.64-0.70, $\mathrm{P}<0.001)$. Older age, male sex, white race, non-academic treatment facility, nonmetropolitan location, stage IIIB, Charlson/Deyo score $>0$ and RT alone were associated with an inferior OS, while the benefit of CRT was greater for patients treated with multi-agent chemotherapy (HR $=0.64,95 \%$ CI, 0.61-0.67, $\mathrm{P}<0.001$ ) compared to single agent chemotherapy (HR $=0.83,95 \%$ CI, 0.75-0.92, $\mathrm{P}<0.001)$. Breaking the CRT group down further, the authors found that the median OS with SCRT was 20.0 months (95\% CI, 19.1-20.9) versus 17.8 months (95\% CI, 17.4-18.2) with CCRT, $\mathrm{P}<0.001$.

These findings support our intuition, that elderly patients who are treated with multi-agent chemotherapy in combination with RT achieve median OS similar to their younger counterparts and survive longer than their peers who receive less intensive therapy. The biggest concern that physicians have with offering this approach is the toxicity profile, which Miller et al. were unable to capture from the database. The other limitations from this study include lack of data on important prognostic factors such as performance status and weight loss, and no details on staging investigations, lung function or co-morbidities. In their discussion, the authors acknowledged that multiple studies have shown that toxicity rates were higher with
CRT compared to RT. This is where careful screening and selection of elderly patients based on tools such as CGA is crucial.

There is a certain level of scepticism amongst oncologists with regards to CRT for stage III NSCLC in the elderly, especially when severe toxicity is encountered, hence clouding one's judgement. The findings of Miller et al. are an important reminder that the risk of not putting suitable patients forward for CRT is an inferior OS. A somewhat intriguing but reassuring finding was that SCRT patients derived a positive survival benefit, and if anything better than the CCRT patients. This finding, discordant to studies in younger patients, may be due to the retrospective nature of the study and an inability to match patients for important characteristics.

This is a particularly relevant discussion because the new standard-of-care for stage III NSCLC is CCRT followed by 12 months of durvalumab. The rationale for introducing the immune checkpoint inhibitor into this treatment paradigm is two-fold, firstly CCRT enhancing tumour immunogenicity and secondly, eradication of micrometastases outside the CRT field. It is unclear if a similar progression-free and OS benefit would be seen if durvalumab was introduced after SCRT or after RT alone. Looking back to the pre-immune checkpoint inhibitor days, an important observation relevant to this discussion comes from the START study, a large phase III study which recruited 1,513 stage III NSCLC patients who had completed CRT to receive tecemotide, a MUC1 antigenspecific immunotherapy, or placebo (30). The study was negative for the primary endpoint of OS. However, the median OS in patients who had received CCRT then tecemotide ( $65 \%$ of patients in the study) was 30.8 months (95\% CI, 25.6-36.8) compared with 20.6 months (95\% CI, 17.4-23.9), HR 0.78, 95\% CI, 0.64-0.95, $\mathrm{P}=0.016$, for placebo, while there was no difference seen in the SCRT group. Therefore, until such time as we have data to support alternate approaches, CRT should be delivered as CCRT prior to durvalumab consolidation.

However, rather than abandoning the proven effective strategy of CCRT then durvalumab in less fit elderly patients, we should build on the PACIFIC trial results. The opportunities for clinical trials might include an abbreviated course of CCRT followed by durvalumab in elderly patients considered unable to tolerate full course treatment, or use of weekly single-agent carboplatin as part of CCRT in elderly patients with poor performance status. Such trials might extend treatment with a high cure rate to elderly patients 
currently treated with RT alone or palliative approaches. Importantly though, given the strength of the data from the PACIFIC study, elderly patients who are deemed fit enough should be considered for CCRT followed by adjuvant durvalumab.

According to the United Nations, longevity is one of the most significant social transformations of the $21^{\text {st }}$ century. This is due to rapid advances in modern medicine with early identification and aggressive management of cardiovascular risk factors and large population based early cancer screening efforts, being two of the key components of this success story. The circumstance of a global ageing population however is increasing rates of cancer, with lung cancer being the leading cause of cancer-related deaths world-wide. As a result, we are going to be faced with an increasing number of elderly stage III NSCLC patients in the clinic. Unique factors which we need to consider in making treatment decisions in this population include performance status, functional lung capacity, nutrition and total body weight loss which are incorporated into the CGA assessment.

Although the PACIFIC study is the most important innovation in stage III NSCLC since the advent of CRT, the field remains ripe for research activity. The opportunities are to investigate strategies to improve efficacy, such as up-front CCRT concurrent with immunotherapy, or development of combination immunotherapy strategies. However, there are also opportunities in elderly patients, who previously would not have been treated with curative intent, to de-escalate therapy while maintaining efficacy. In essence, Miller et al. have confirmed an instinctive thought, that in a real-world scenario, maximising therapy by adding in chemotherapy to RT in elderly stage III NSCLC patients results in a significant OS advantage compared to RT alone. Their findings are instructive and serve us a reminder that with careful patient selection, 'fortune favours the bold'.

\section{Acknowledgements}

Dr. Surein Arulananda received a La Trobe University Post Graduate Research Scholarship.

\section{Footnote}

Conflicts of Interest: Paul Mitchell declares Advisory BoardsAstraZeneca, Roche, Boehringer-Ingelheim, BMS, MSD, Celgene; Honoraria-Roche, Merck KGa; Travel Grants (conferences)-Roche, BMS, AstraZeneca. Dr. Surein Arulananda has no conflicts of interest to declare.

\section{References}

1. National Cancer Institute: SEER stat fact sheets: Lung and bronchus cancer. Available online: https://seer.cancer. gov/statfacts/html/lungb.html. Accessed 28 July 2018.

2. Balducci L. Geriatric oncology: challenges for the new century. Eur J Cancer 2000;36:1741-54.

3. Miller ED, Fisher JL, Haglund KE, et al. The Addition of Chemotherapy to Radiation Therapy Improves Survival in Elderly Patients with Stage III Non-Small Cell Lung Cancer. J Thorac Oncol 2018;13:426-35.

4. Schaake-Koning C, van den Bogaert W, Dalesio O, et al. Effects of concomitant cisplatin and radiotherapy on inoperable non-small-cell lung cancer. N Engl J Med 1992;326:524-30.

5. Lee JS, Scott C, Komaki R, et al. Concurrent chemoradiation therapy with oral etoposide and cisplatin for locally advanced inoperable non-small-cell lung cancer: radiation therapy oncology group protocol 91-06. J Clin Oncol 1996;14:1055-64.

6. Dillman RO, Seagren SL, Propert KJ, et al. A randomized trial of induction chemotherapy plus high-dose radiation versus radiation alone in stage III non-small-cell lung cancer. N Engl J Med 1990;323:940-5.

7. Curran WJ Jr, Paulus R, Langer CJ, et al. Sequential vs. concurrent chemoradiation for stage III non-small cell lung cancer: randomized phase III trial RTOG 9410. J Natl Cancer Inst 2011;103:1452-60.

8. Auperin A, Le Pechoux C, Rolland E, et al. Meta-analysis of concomitant versus sequential radiochemotherapy in locally advanced non-small-cell lung cancer. J Clin Oncol 2010;28:2181-90.

9. Rusch VW, Giroux DJ, Kraut MJ, et al. Induction chemoradiation and surgical resection for superior sulcus non-small-cell lung carcinomas: long-term results of Southwest Oncology Group Trial 9416 (Intergroup Trial 0160). J Clin Oncol 2007;25:313-8.

10. Albain KS, Crowley JJ, Turrisi AT 3rd, et al. Concurrent cisplatin, etoposide, and chest radiotherapy in pathologic stage IIIB non-small-cell lung cancer: a Southwest Oncology Group phase II study, SWOG 9019. J Clin Oncol 2002;20:3454-60.

11. Socinski MA, Rosenman JG, Halle J, et al. Dose-escalating conformal thoracic radiation therapy with induction and concurrent carboplatin/paclitaxel in unresectable stage IIIA/B nonsmall cell lung carcinoma: a modified phase I/II trial. Cancer 2001;92:1213-23.

12. Yamamoto N, Nakagawa K, Nishimura Y, et al. Phase III study comparing second- and third-generation regimens 
with concurrent thoracic radiotherapy in patients with unresectable stage III non-small-cell lung cancer: West Japan Thoracic Oncology Group WJTOG0105. J Clin Oncol 2010;28:3739-45.

13. Senan S, Brade A, Wang LH, et al. PROCLAIM: Randomized Phase III Trial of Pemetrexed-Cisplatin or Etoposide-Cisplatin Plus Thoracic Radiation Therapy Followed by Consolidation Chemotherapy in Locally Advanced Nonsquamous Non-Small-Cell Lung Cancer. J Clin Oncol 2016;34:953-62.

14. Liang J, Bi N, Wu S, et al. Etoposide and cisplatin versus paclitaxel and carboplatin with concurrent thoracic radiotherapy in unresectable stage III non-small cell lung cancer: a multicenter randomized phase III trial. Ann Oncol 2017;28:777-83.

15. Liew MS, Sia J, Starmans MH, et al. Comparison of toxicity and outcomes of concurrent radiotherapy with carboplatin/paclitaxel or cisplatin/etoposide in stage III non-small cell lung cancer. Cancer Med 2013;2:916-24.

16. Tsujino K, Kurata T, Yamamoto S, et al. Is consolidation chemotherapy after concurrent chemo-radiotherapy beneficial for patients with locally advanced non-small-cell lung cancer? A pooled analysis of the literature. J Thorac Oncol 2013;8:1181-9.

17. Vokes EE, Herndon JE 2nd, Kelley MJ, et al. Induction chemotherapy followed by chemoradiotherapy compared with chemoradiotherapy alone for regionally advanced unresectable stage III Non-small-cell lung cancer: Cancer and Leukemia Group B. J Clin Oncol 2007;25:1698-704.

18. Gore E. RTOG 0214: a phase III comparison of prophylactic cranial irradiation versus observation in patients with locally advanced non-small cell lung cancer. Clin Adv Hematol Oncol 2005;3:625-6.

19. Bradley JD, Paulus R, Komaki R, et al. Standard-dose versus high-dose conformal radiotherapy with concurrent and consolidation carboplatin plus paclitaxel with or without cetuximab for patients with stage IIIA or IIIB non-smallcell lung cancer (RTOG 0617): a randomised, two-by-two factorial phase 3 study. Lancet Oncol 2015;16:187-99.

20. Antonia SJ, Villegas A, Daniel D, et al. Durvalumab after Chemoradiotherapy in Stage III Non-Small-Cell Lung Cancer. N Engl J Med 2017;377:1919-29.

21. British Geriatrics Society. Comprehensive Assessment of the Frail Older Patient. Available online: http://www.bgs. org.uk/cga-managing/resources/campaigns/fit-for-frailty/ frailty-cga. Accessed 28th July 2018.

22. Hayakawa K, Mitsuhashi N, Katano S, et al. High-dose radiation therapy for elderly patients with inoperable or unresectable non-small cell lung cancer. Lung Cancer 2001;32:81-8.

23. Schild SE, Stella PJ, Geyer SM, et al. The outcome of combined-modality therapy for stage III non-small-cell lung cancer in the elderly. J Clin Oncol 2003;21:3201-6.

24. Jalal SI, Riggs HD, Melnyk A, et al. Updated survival and outcomes for older adults with inoperable stage III nonsmall-cell lung cancer treated with cisplatin, etoposide, and concurrent chest radiation with or without consolidation docetaxel: analysis of a phase III trial from the Hoosier Oncology Group (HOG) and US Oncology. Ann Oncol 2012;23:1730-8.

25. Langer CJ, Hsu C, Curran WJ, et al. Elderly patients (pts) with locally advanced non-small cell lung cancer (LANSCLC) benefit from combined modality therapy. secondary analysis of Radiation Therapy Oncology Group (RTOG) 94-10. Proc Am Soc Clin Oncol 2002;21:Abstr 1193.

26. Rocha Lima CM, Herndon JE, Kosty M et al. Therapy choices among older patients with lung carcinoma: an evaluation of two trials of the Cancer and Leukemia Group B. Cancer 2002;94:181-7.

27. Atagi S, Kawahara M, Yokoyama A, et al. Thoracic radiotherapy with or without daily low-dose carboplatin in elderly patients with non-small-cell lung cancer: a randomised, controlled, phase 3 trial by the Japan Clinical Oncology Group (JCOG0301). Lancet Oncol 2012;13:671-8.

28. Clamon G, Herndon J, Cooper R, et al. Radiosensitization with carboplatin for patients with unresectable stage III non-small-cell lung cancer: a phase III trial of the Cancer and Leukemia Group B and the Eastern Cooperative Oncology Group. J Clin Oncol 1999;17:4-11.

29. Pallis AG, Gridelli C, van Meerbeeck JP, et al. EORTC Elderly Task Force and Lung Cancer Group and International Society for Geriatric Oncology (SIOG) experts' opinion for the treatment of non-small-cell lung cancer in an elderly population. Ann Oncol 2010;21:692-706.

30. Butts C, Socinski MA, Mitchell PL, et al. Tecemotide (L-BLP25) versus placebo after chemoradiotherapy for stage III non-small-cell lung cancer (START): a randomised, double-blind, phase 3 trial. Lancet Oncol 2014;15:59-68.

Cite this article as: Arulananda S, Mitchell P. Elderly patients with stage III NSCLC survive longer when chemotherapy is added to radiotherapy-fortune favours the bold. Transl Lung Cancer Res 2018;7(Suppl 4):S388-S392. doi: 10.21037/ tlcr.2018.08.12 